\title{
ASSOCIATION BETWEEN LENGTH OF STORAGE OF ERYTHROCYTES AND POSTOPERATIVE ACUTE RENAL DYSFUNCTION IN PATIENTS UNDERGOING RE-OPERATIVE CARDIAC SURGERY
}

\section{Sukhjeewan Basran, Robert Frumento, Allison Cohen, Sam Lee, Ervant Nishanian}

Department of Anesthesiology, Columbia University College of Physicians \& Surgeons, 630 West $168^{\text {th }}$ St. - PH505, New York, NY, 10032-3784

INTRODUCTION: Acute renal failure (ARF) requiring dialysis occurs in approximately 1-3\% of patients after major cardiovascular surgery. ${ }^{1}$ Acute renal dysfunction (ARD) occurs in approximately $8 \%$ of patients after major cardiovascular surgery. ${ }^{2}$ The Transfusion Requirements in Critical Care study raised the possibility that during critical illness the transfusion of stored red blood cells may have adverse effects. ${ }^{3}$ These effects may be due to the storage time depletion of red cell 2, 3 DPG and ATP and reduced red cell deformability. This storage lesion could impair oxygen delivery to renal tissues by reducing both capillary flow and oxygen unloading from hemoglobin.

METHODS: This was a single-center, retrospective cohort study of patients undergoing reoperation between the years $1996 \& 2002$. Transfusion data was collected through postoperative day 7 .

Exclusion criteria were preoperative dialysis, preoperative creatinine value $\geq 2 \mathrm{mg} / \mathrm{dl}$ and patients who received irradiated blood transfusions. All demographic and outcome variables related to the study were collected by an investigator who was blinded to the duration of RBC storage data. Variables used to study the effect of storage duration for each patient were 1) the mean duration of storage of all RBCs transfused and 2) duration of storage of the oldest and youngest unit transfused. Acute renal dysfunction was defined as a serum creatinine increase postoperatively to $\geq 2 \mathrm{mg} / \mathrm{dL}$ with an increase of $\geq 0.7 \mathrm{mg} / \mathrm{dL}$ within 2 weeks after surgery OR Need for dialysis within 2 weeks after surgery. ${ }^{2}$ RESULTS: 434 patients were evaluated and 297 met inclusion criteria. 285/297(96\%) patients received $\geq 2$ units of allogeneic erythrocytes. $52(17.5 \%)$ patients developed postoperative ARD and 5 of these $52(9.6 \%)$ required postoperative dialysis. The mean length of storage of all transfused RBCs to each patient was greater among patients developing ARD than in those who did not (table 1). Similarly, the mean length of storage of the oldest and the youngest RBCs transfused and the total number of RBCs transfused was greater among patients developing ARD (table 1). Univariate predictors of postoperative ARD (aortic cross clamp time, cardiopulmonary bypass time, preoperative creatinine, age, number of units transfused) were tested for entry into a multivariate logistic regression model predicting the development of ARD. The total number of RBCs transfused was a no longer a significant predictor $(\mathrm{p}=0.2)$ of postoperative ARD. However, the mean, minimum and maximal age of transfused erythrocytes remained significant in the univariate analysis.

CONCLUSION: Patients undergoing cardiac surgical reoperations are at risk for the development of postoperative acute renal dysfunction. We report an association between the length of storage of perioperatively administered RBCs and the frequency of postoperative ARD in this patient population. Whether this association is causative and independent of unknown factors should be established in prospective randomized trial.

Table 1 - Length of Storage of Erythrocytes and Postoperative Acute Renal Dysfunction

\begin{tabular}{lcccc}
\hline Acute Renal Dysfunction & \# PRBC Units & \multicolumn{3}{c}{ Length of Storage } \\
\hline & & $\underline{\text { All }}$ & $\underline{\text { Oldest }}$ & Youngest \\
YES: $52(17.5 \%)$ & $8.6 \pm 8.9$ & $24.9 \pm 5.2$ & $29.0 \pm 6.2$ & $21.9 \pm 6.2$ \\
NO: $245(82.5 \%)$ & $5.0 \pm 4.6$ & $20.8 \pm 5.2$ & $24.2 \pm 7.9$ & $17.3 \pm 6.8$ \\
p Value & $<0.0001$ & $<0.0001$ & $<0.0001$ & $<0.0001$ \\
\hline
\end{tabular}

1. Nephrol Dial Transplant 1999; 14: 1158-62

2. Ann Intern Med 1998; 128: 194-203

3. N Engl J Med 1999; 340: 409-17 